\title{
Myocardial contrast echocardiography in the diagnosis of a left ventricular hemangioma
}

\author{
Kyriacos Papadopoulos, ${ }^{1}$ Constantinos Makrides ${ }^{2}$ \\ ${ }^{1}$ Medical Check-Up Center, Nicosia; ${ }^{2}$ General Practitioner, Limassol, Cyprus
}

\begin{abstract}
Myocardial perfusion contrast echocardiography is an imaging tool for the assessment of myocardial microcirculation. It can also be used for the evaluation of the relative perfusion of a cardiac mass. Cardiac hemangiomas are rare tumors, accounting for only $2.8 \%$ of all benign primary cardiac tumors. We report a case of a 17 -year-old man with a left ventricular hemangioma detected with transthoracic echocardiography. Myocardial perfusion contrast echocardiography was used to evaluate the vascularity of the cardiac mass. Using this technique malignant and hypervascular (e.g., hemangioma) tumors display persistence of contrast enhancement compared to the surrounding myocardium. The mass was successfully removed and the final report from pathology showed that the left ventricular mass was a hemangioma, a hypervascular tumor.
\end{abstract}

\section{Introduction}

Cardiac hemangiomas are extremely rare forms of tumor and account for only $2.8-5 \%$ of all benign cardiac tumours. ${ }^{1,2}$

Two-dimensional echocardiography is regarded as the primary diagnostic imaging technique for the evaluation of cardiac mass lesions, with diagnostic sensitivity of $93 \%$ for transthoracic echocardiography and $97 \%$ for transesophageal echocardiography. ${ }^{3}$

Myocardial contrast echocardiography provides additional information about the perfusion of cardiac masses and differentiates the neo-vascularization of a

Correspondence: Kyriacos Papadopoulos, Medical Check-Up Center, 21 st Costa Anaxagora steet, Nicosia, Cyprus.

Tel.: +35.799460314 - Fax: +35.722499150.

E-mail: kyriacospap@hotmail.com

Key words: Contrast echocardiography; myocardial microcirculation; hemangioma; heart neoplasms; left ventricle; hypervascular tumor.

Contributions: the authors contributed equally.

Conflict of interest: the authors declare no potential conflict of interest.

Received for publication: 26 June 2015.

Accepted for publication: 18 August 2015.

This work is licensed under a Creative Commons Attribution NonCommercial 4.0 License (CC BY-NC 4.0).

CCopyright K. Papadopoulos and C. Makrides, 2016

Licensee PAGEPress, Italy

Italian Journal of Medicine 2016; 10:151-153

doi:10.4081/itjm.2016.618 malignancy or vascular tumor from the avascularity of a thrombus and the sparse vascularity of a benign stromal tumor., ${ }^{4,5}$

We describe a patient with a hemangioma of the left ventricle discovered during transthoracic echocardiography. Myocardial contrast echocardiography revealed the vascular nature of the tumor, affording an important clue to the diagnosis of cardiac hemangioma.

\section{Case Report}

A 17-year-old man was referred to a Cardiologist for evaluation of a systolic murmur. His past medical history was unremarkable. Physical examination revealed a blood pressure of $125 / 80 \mathrm{mmHg}$ with a regular pulse of 77 beats/min. Cardiac auscultation revealed normal first and second heart sounds and a systolic murmur $2 / 6$, best heard along the cardiac apex. Lung sounds were clear.

An electrocardiogram showed normal sinus rhythm, normal axis and no ST segment or T wave changes. His chest X-ray showed no obvious abnormality. Routine blood investigations were normal. Transthoracic echocardiogram showed a normal left ventricular ejection fraction and an echogenic mass in the left ventricle. The mass was located on the lateral wall of the left ventricle, attached on the anterolateral papillary muscle (Figure 1, Video 1). Mild mitral regurgitation was also present, explaining the origin of the systolic murmur.

We then used myocardial contrast echocardiography to evaluate the vascularity of the cardiac mass. After a $0.2 \mathrm{~mL}$ intravenous injection of sulphur hexafluoride, an ultrasound impulse of high-mechanical index was transmitted to destroy microbubbles within the mass. 
The mass was rapidly filled with contrast greater than the surrounding myocardium, suggesting an intense vascularization (Figure 2, Video 2).

The patient was referred for elective surgical excision of the mass because of its potential for embolization and possible malignancy.

The surgical procedure was performed under cardiopulmonary bypass support. The postoperative course was uneventful, without any complications.

The histopathology study showed cardiac hemangioma.

\section{Discussion}

Myocardial contrast echocardiography allows the assessment of myocardial perfusion by imaging the coronary microcirculation. Several studies have shown that myocardial contrast echocardiography is a feasible and accurate method for the evaluation of ischemic heart disease. This includes both diagnostic and prognostic evaluation.

Myocardial contrast echocardiography uses microbubbles that are inert, remain entirely within the vascular space and have similar rheology to red blood cells ${ }^{6,7}$ The patients receive an injection of a contrast agent directly into the bloodstream through a vein. High energy pulse of ultrasound is used to destroy the microbubbles and observation of the subsequent replenishment of microbubbles into the microcirculation permits evaluation of myocardial tissue perfusion. ${ }^{8}$ Normally it takes $5 \mathrm{~s}$ for complete replenishment of the myocardium. Any decrease in the myocardial blood flow prolongs the replenishment time in proportion to the reduction in myocardial blood flow. ${ }^{8}$ At hyperemic flows the replenishment is faster.
Myocardial perfusion contrast echocardiography can also be used to help us characterize the vascularity of cardiac masses.

Myocardial tissue is highly vascular (capillary density on the order of 2500 to $3000 / \mathrm{mm}^{3}$ ). ${ }^{9}$ The total blood carrying capacity of the abnormal dilated vessels of the malignant tumors and of the vessels of vascular tumors (e.g., hemangioma) is probable to be in excess of that of the adjacent myocardium. Stromal tumors have lower vascularization and thrombi are avascular.

Quantification of echocardiographic perfusion imaging of cardiac masses facilitates the differential diagnosis between different types of cardiac masses.

Malignant or highly vascular tumors demonstrate greater contrast enhancement than the adjacent myocardium. Stromal tumors display partial enhance-

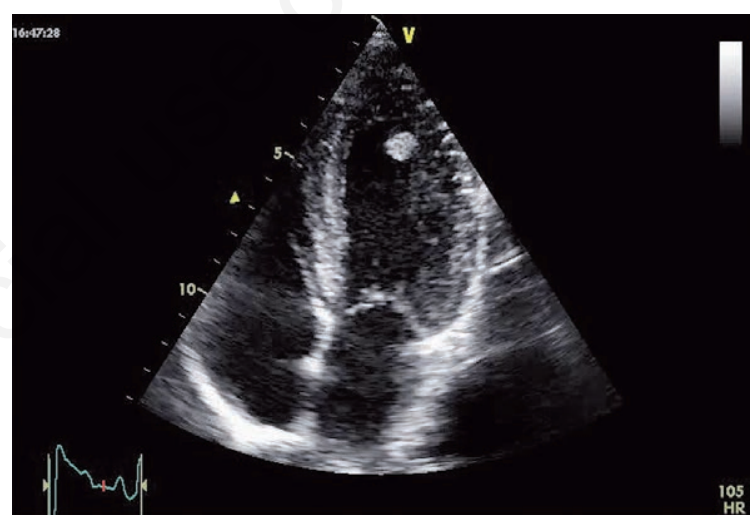

Figure 1. Transthoracic echocardiography revealed an echodense mass in the left ventricle (four chamber view) [see also online Video 1].
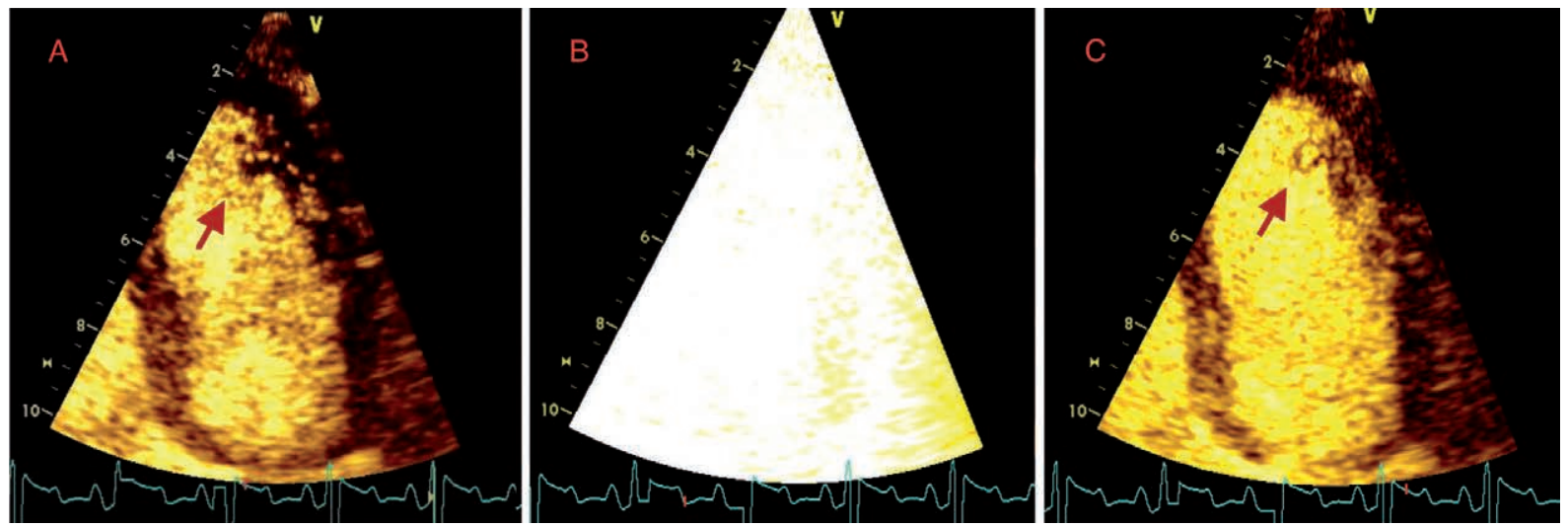

Figure 2. Myocardial contrast echocardiography demonstrated greater contrast enhancement of the mass than the surrounding myocardium, identifying its vascular nature. A) Baseline; B) contrast destruction; C) contrast replenishment [see also online Video 2]. 
ment and less perfusion than the adjacent myocardium and thrombi demonstrate a complete lack of contrast enhancement.

Therefore echocardiographic contrast perfusion imaging may aid in the early identification of the type of the cardiac mass in order to select the best possible treatment strategy. Malignant primary cardiac tumors are potentially curable with surgical resection if discovered early. Identification of a cardiac thrombus would be an indication for anticoagulation with a follow up study to evaluate resolution.

In our case myocardial perfusion contrast echocardiography demonstrated greater contrast enhancement of the mass than the adjacent myocardium, identifying its vascular nature. Tissue pathological study showed that the left ventricular mass was a hemangioma, a hypervascular tumor.

Hemangioma of the heart, presenting as a primary cardiac tumor is extremely rare; it accounts for approximately $2 \%$ of all primary resected heart tumors. They can occur at any age and can be localized in any part of the heart and pericardium.

Though most cardiac hemangiomas are asymptomatic and discovered incidentally, they may cause arrhythmia, pericardial effusion, coronary insufficiency, left or right ventricular outflow tract obstruction, embolization or congestive heart failure and sudden cardiac death. ${ }^{10}$ Diagnosis can be made by echocardiography, computed tomography, or magnetic resonance imaging.

As the natural history of cardiac hemangioma is unpredictable, surgery is the appropriate treatment. $\mathrm{Pa}$ tients with a resectable tumor usually have a good prognosis, but those with an unresectable tumor may have a poor prognosis because of ventricular tachycardia, sudden death, local progression, or systemic dissemination of the malignant tumor.

This report underlines the importance of the echocardiography as an accurate, non-invasive imaging modality for detecting cardiac tumours, including cardiac hemangioma. However, preoperative diagnosis of cardiac hemangioma is notoriously elusive. In our case, myocardial contrast echocardiography identified the vascular nature of the tumor and differentiated it from other relatively avascular structures such as myxomas, lipomas and fibromas, affording an important clue to the diagnosis of cardiac hemangioma.

\section{Conclusions}

We can conclude the following: i) echocardiography is a sensitive non-invasive method for detecting the cardiac hemangioma initially; ii) further characterization of the tumor can be made using myocardial contrast echocardiography which can demonstrate the vascularity of the mass; iii) myocardial perfusion contrast echocardiography appears to be a valuable diagnostic tool in differentiating the different types of cardiac masses.

\section{References}

1. McAllister HA. Tumors of the heart and pericardium. In: Silver MD, ed. Cardiovascular pathology. New York, NY: Churchill Livingstone; 1983. pp 909-43.

2. Serri K, Schraub P, Lafitte S, Roudaut R. Cardiac hemangiomas presenting as atypical chest pain. Eur $\mathrm{J}$ Echocardiogr 2007;8:17-8.

3. Meng Q, Lai H, Lima J, et al. Echocardiographic and pathologic characteristics of primary cardiac tumors: a study of 149 cases. Int J Cardiol 2002;84:69-75.

4. Haverkamp MC, Scholte AJ, Holman ER, et al. Contrast echocardiography as a useful additional diagnostic tool in evaluating a primary cardiac tumor. Eur J Echocardiogr 2005;6:388-91.

5. Kirkpatrick JN, Wong T, Bednarz JE, et al. Differential diagnosis of cardiac masses using contrast echocardiographic perfusion imaging. J Am Coll Cardiol 2004;43: 1412-9.

6. Jayaweera AR, Edwards N, Glasheen WP, et al. In vivo myocardial kinetics of air-filled albumin microbubbles during myocardial contrast echocardiography: comparison with radio-labeled red blood cells. Circ Res 1994;74:1157-65.

7. Keller MW, Segal SS, Kaul S, Duling B. The behaviour of sonicated albumin microbubbles within the microcirculation: a basis for their use duringmyocardial contrast echocardiography. Circ Res 1989;65:458-67.

8. Wei K, Jayaweera AR, Firoozan S, et al. Quantification of MBF flow with ultrasound-induced destruction of microbubbles administered as a constant venous infusion. Circulation 1998;97:473-83.

9. Le DE, Bin JP, Coggins MP, et al. Relation between myocardial oxygen consumption and myocardial blood volume: a study using myocardial contrast echocardiography. J Am Soc Echocardiogr 2002;15:857-63.

10. Kojima S, Sumiyoshi M, Suwa S, et al. Cardiac haemangioma: a report of two cases and review of the literature. Heart Vessels 2003;18:153-6. 